\title{
Dynamic Bertrand and Cournot Competition: Asymptotic and Computational Analysis of Product Differentiation
}

\author{
Andrew Ledvina* $\quad$ Ronnie Sircar ${ }^{\dagger}$
}

February 14, 2011

\begin{abstract}
We study continuous time oligopolies in which a small number of firms producing similar goods compete with one another by setting prices or quantities. We study a deterministic version of the problem using an asymptotic expansion of the relevant HJB partial differential equations. We find in this setting that for firms with highly differentiated goods, the type of competition matters little. For less differentiated goods, we find that the Cournot type market produces a greater value to the firms than the Bertrand type market. We then study a stochastic version of the two games using numerical techniques. This allows us to compare firms with a greater degree of product differentiation. The value is still greater to firms in most scenarios in a Cournot market, but in some situations the classical Bertrand-Cournot dichotomy is reversed.
\end{abstract}

\section{Introduction}

Oligopolistic competition has been studied extensively in the economic literature, beginning with Cournot [1838]. In this original work, firms compete with one another in a static set-up by choosing quantities to supply of a homogeneous good. This was later criticized in Bertrand [1883] as it was said firms actually compete by setting prices. Models where firms set prices are now generally referred to as being of Bertrand type and models where firms set quantities are termed as being of Cournot type. Our intention is to study price-setting and quantity-setting oligopolies in continuoustime and where the goods are differentiated from one another. However, much of the intuition about what one expects in certain market types is still grounded in the original static models. For example, the original Bertrand model results in perfect competition in all cases besides monopoly, which is unrealistic in most settings, leading one to conclude that the correct set-up leads to the wrong result. The Cournot model leads to more realistic outcomes, but as most firms seem to set their prices, not their quantities, many economists have argued that the Cournot model gives the right answer for the wrong reason.

*ORFE Department, Princeton University, Sherrerd Hall, Princeton NJ 08544; aledvina@princeton.edu. Work partially supported by NSF grant DMS-0739195.

${ }^{\dagger}$ ORFE Department, Princeton University, Sherrerd Hall, Princeton NJ 08544; sircar@princeton.edu. Work partially supported by NSF grant DMS-0807440. 
While Bertrand and Cournot considered homogeneous goods, we will consider differentiated goods, in particular substitute goods. In other words, consumption of one good can be substituted for another, but not perfectly. The original work in this area is Hotelling [1929], where consumers were assumed to associate a cost of travel depending on the location of the firms, thereby differentiating their otherwise identical goods based on the location of the firms relative to the location of the consumer. For classical results on differentiated goods and other models of oligopoly, we refer to the books by Friedman [1983] and Vives [1999]. We shall see that the fundamental ingredient to our comparison is how similar the goods of the firms are to one another.

Our objective is to study the effect of product differentiation on the outcomes in these two oligopoly models. This builds on an earlier analysis of nonzero-sum differential games of Bertrand type in Ledvina and Sircar [2011] and Cournot type in Harris et al. [2010]. Moreover, this work is complementary to our comparison of Bertrand and Cournot oligopolies in a static setting in Ledvina and Sircar [2010]. Here we compare Bertrand and Cournot oligopolies in a continuous-time framework.

\section{Oligopoly Markets for Differentiated Goods}

As our stated purpose is to concentrate on the role of product differentiation, we shall focus on duopolies facing linear demand. This already is of sufficient complexity to present computational and analytical challenges, and to reveal important effects of dynamic competition. In addition, we shall consider both stochastic and non-stochastic demand depending on whether product differentiation is "large" or "small". The intention of this section is first to establish the demand framework which allows us to meaningfully compare Bertrand and Cournot competition. In Section 3, we shall describe the dynamics of the state variables of the firms and introduce the notation associated with the game.

\subsection{Systems of Demand}

We focus on linear demand and therefore we could simply posit a linear demand function that the firms face. However, in order to ensure consistency within the system of demand and between demand and inverse demand, we assume that there exists a representative consumer with a concave utility function $U(q): \mathbb{R}_{+}^{2} \rightarrow \mathbb{R}$ which represents his utility from consuming the quantities $q=$ $\left(q^{1}, q^{2}\right)$ of goods from the firms. Our representative consumer solves the utility maximization (minus cost) problem

$$
\max _{q \in \mathbb{R}_{+}^{2}} U(q)-\sum_{i=1}^{2} p_{i} q_{i},
$$

from which we can derive the inverse demand functions for the firms as

$$
p_{i}(q)=\frac{\partial U}{\partial q_{i}}, \quad i=1,2 .
$$

This gives actual quantities provided $p_{i}>0$ for all $i$. In the case that the quantities result in any of the prices not being positive, we must remove the individual firm with the highest quantity from the system and then consider a market with only one firm. If the remaining firm has a price which is still not positive, then neither firm receives any demand. We then define the direct demand of the 
firms by inverting this system of inverse demands. Therefore, we must assume the utility function is strictly concave which will ensure that the system is invertible.

In order to derive a linear system of demand, we consider a quadratic utility function,

$$
U(q)=\alpha\left(q_{1}+q_{2}\right)-\frac{1}{2} \beta\left(q_{1}^{2}+q_{2}^{2}+2 \varepsilon q_{1} q_{2}\right), \quad \alpha, \beta>0 .
$$

The parameter $\varepsilon$ can be negative for complementary goods, or positive for substitute goods. We shall take it to be positive as the case of substitute goods is a more natural setting in which to discuss competition. We also require $\varepsilon<1$ for the resulting system of inverse demands to be invertible. This is not the most general quadratic utility function that leads to linear demand, however the symmetries that are implicitly assumed in this utility function are not particularly restrictive if one considers asymmetric costs, as will naturally arise in what follows.

Solving (1) results in the inverse demand system

$$
p_{i}(q)=\frac{\partial U}{\partial q_{i}}=\alpha-\beta\left(q_{i}+\varepsilon q_{j}\right), \quad i=1,2 ; j \neq i .
$$

We can invert this system to obtain the duopoly demand

$$
q_{i}(p)=\left(\frac{\alpha}{\beta(1+\varepsilon)}\right)-\left(\frac{1}{\beta\left(1-\varepsilon^{2}\right)}\right)\left(p_{i}-\varepsilon p_{j}\right), \quad i=1,2 ; i \neq j
$$

The associated monopoly inverse demand is given by

$$
p_{m}\left(q_{i}\right)=\alpha-\beta q_{i}
$$

and the resulting direct demand is given by

$$
q_{m}\left(p_{i}\right)=\frac{1}{\beta}\left(\alpha-p_{i}\right) .
$$

The degree of product differentiation is measured by the quantity $\varepsilon \in[0,1)$. If $\varepsilon=0$ then the individual firm inverse demand and direct demand functions are equal to their monopoly counterparts, i.e. each firm has a monopoly in the market for their individual good which implies their behavior is independent of the other firm. At the other end of the spectrum, i.e. the limit as $\varepsilon \rightarrow 1$, we obtain homogeneous goods. This means that the firms are identical to consumers and we obtain the demand functions considered in the classical Cournot and Bertrand papers. We do not consider this limit here, and we shall thus stay within $\varepsilon<1$.

Other systems of demand are discussed in Vives [1999], in [Ledvina and Sircar, 2011, Section 2] for differentiated Bertrand and [Harris et al., 2010, Section 2] for homogeneous Cournot.

\subsection{Welfare and Consumer Surplus}

There are several different ways in which one could compare different market types. For example, all else equal, one could consider the average price charged to consumers or the total quantity

supplied to the market. Every performance metric has both positive and negative attributes and 
therefore it can sometimes be difficult to decide which one is the most meaningful. We shall focus on consumer surplus and economic welfare as our main points of comparison because these have become standard in much of the economic literature, see for example [Mas-Colell et al., 1995, Section 10.E]. However, we will also compare prices and quantities where suitable.

Definition 2.1. Consumer Surplus in the linear oligopoly model is defined as

$$
C S=\frac{1}{2} \sum_{i=1}^{N}\left(\alpha-p_{i}\right) \cdot q_{i} .
$$

See for instance Cellini et al. [2004] and Mukherjee [2005]. If we are in a Bertrand market then $p_{i}$ is controlled by Firm $i$ and $q_{i}$ is determined through the demand function. Contrarily, in a Cournot market, $q_{i}$ is controlled by Firm $i$ and $p_{i}$ is determined through the inverse demand function.

Definition 2.2. Economic Welfare is the sum of consumer surplus and aggregate market profits (up to a constant which is irrelevant when comparing differences between welfare in two game types):

$$
E W=C S+\sum_{i=1}^{N} \pi_{i}
$$

where $\pi_{i}$ is the profit of Firm $i$.

There exist alternative definitions using the utility function of the consumers, but for our purposes the above definitions are sufficient. In Section 5.2, we compare dynamic Cournot and Bertrand games using these measures.

\section{Dynamic Game Formulation}

The dynamic games that we study are ones where the capacities of the firms are running out, and when one has exhausted its resources, it no longer participates and the other firm has a monopoly until it also has used up its capacity. In the Bertrand model in Ledvina and Sircar [2011], the state variables were the capacities of two firms producing differentiated consumer goods used to measure their relative size. In Harris et al. [2010], the state variables were the firms' reserves of a homogeneous exhaustible resource such as oil.

\subsection{Dynamic Oligopolies}

Denote the capacity of Firm $i$ by $X_{t}^{i, b}, X_{t}^{i, c}$ for the Bertrand and Cournot games, respectively. Throughout what follows a superscript $b$ will indicate a variable related to the game of Bertrand type and a superscript $c$ for Cournot. We look for Markov Perfect equilibria, in other words firms use Markovian strategies. In the Bertrand game, let the Markovian price strategy of Firm $i$ at time $t$ be given by $p^{i}\left(X_{t}^{1, b}, X_{t}^{2, b}\right), i=1,2$. Similarly, in the Cournot game, let the Markovian strategic rate of supply of Firm $i$ be given by $q^{i}\left(X_{t}^{1, c}, X_{t}^{2, c}\right)$. In a game of Bertrand type, as price, $p^{i}$, is the control variable (for which the firm number is superscripted), the rate of demand is given by the direct demand function, $q_{i}$, defined in (5) (for which the firm number is subscripted). In a game 
of Cournot type where the rate of supply, $q^{i}$, is the control variable (for which the firm number is superscripted), price is given by the inverse demand function, $p_{i}$, defined in (4) (for which the firm number is subscripted).

In the Bertrand game, the dynamics of the state processes are given by the controlled stochastic differential equations

$$
d X_{t}^{i, b}=-q_{i}\left(p^{1}\left(X_{t}^{1, b}, X_{t}^{2, b}\right), p^{2}\left(X_{t}^{1, b}, X_{t}^{2, b}\right)\right) d t+\sigma^{i} d W_{t}^{i}
$$

for $i=1,2$ and where $\left(W_{t}^{1}\right)$ and $\left(W_{t}^{2}\right)$ are correlated Brownian motions with

$$
\mathbb{E}\left\{d W_{t}^{1} \cdot d W_{t}^{2}\right\}=\rho d t
$$

These are the correct dynamics provided $X_{t}^{1, b}>0, X_{t}^{2, b}>0$. If either one is strictly positive and the other is zero, then we replace the $q_{i}$ corresponding to the positive quantity with $q_{m}$ and the quantity that hits zero remains at zero thereafter. In other words if there is some $t$ such that $X_{t}^{i, b}=0$ then $X_{s}^{i, b}=0$ for all $s \geq t$. We further assume that $X_{0}^{i, b}$ are known fixed positive constants.

The dynamics for the Cournot state variables are defined in a similar fashion. Namely,

$$
d X_{t}^{i, c}=-q^{i}\left(X_{t}^{1, c}, X_{t}^{2, c}\right) d t+\sigma^{i} d W_{t}^{i}
$$

where the Brownian motions are the same as those above. Again, these only hold for $X_{t}^{1, c}>$ $0, X_{t}^{2, c}>0$, but here things are slightly more simple. If either state variable hits zero then the corresponding $q^{i}$ is equal to zero. Therefore, we only need to stipulate that if there exists $t$ such that $X_{t}^{i, c}=0$ then $X_{s}^{i, c}=0$ for all $s \geq t$. In other words, the diffusion cannot resurrect a firm once it has run out of capacity.

The Brownian motions in (10) and (12) could represent uncertainty of actual demand or of remaining reserves depending on the context of actual application.

\subsection{Nash Equilibrium and the HJB PDEs}

The objective of the firms is to maximize expected lifetime profit in an equilibrium sense to be made precise below. To this end, we define the profit functionals of the firms

$$
\begin{aligned}
& J^{i, b}\left(p^{1}\left(x_{1}, x_{2}\right), p^{2}\left(x_{1}, x_{2}\right)\right) \triangleq \mathbb{E}_{x_{1}, x_{2}}\left\{\int_{0}^{\tau^{i, b}} e^{-r t} p^{i} q_{i}\left(p^{1}, p^{2}\right) d t\right\}, \\
& J^{i, c}\left(q^{1}\left(x_{1}, x_{2}\right), q^{2}\left(x_{1}, x_{2}\right)\right) \triangleq \mathbb{E}_{x_{1}, x_{2}}\left\{\int_{0}^{\tau^{i, c}} e^{-r t} p_{i}\left(q^{1}, q^{2}\right) q^{i} d t\right\},
\end{aligned}
$$

where $\tau^{i, b}=\inf \left\{t>0: X_{t}^{i, b}=0\right\}$, and similarly for $\tau^{i, c}$. Further,

$$
\mathbb{E}_{x_{1}, x_{2}}\{\cdot\}=\mathbb{E}\left\{\cdot \mid X_{0}^{i, \cdot}=x_{1}, X_{0}^{i, \cdot}=x_{2}\right\}
$$

with the appropriate state variables understood by context, and $r>0$ is a discount rate. 
Definition 3.1. A vector $p^{\star}$ is a Markov Perfect Nash equilibrium of the dynamic Bertrand game if for all positive and suitably regular (for example Lipschitz, see Başar and Olsder [1995]) Markov controls $p^{1}$ we have

$$
J^{1, b}\left(p^{1, \star}, p^{2, \star}\right) \geq J^{1, b}\left(p^{1}, p^{2, \star}\right),
$$

and for all such Markov controls $p^{2}$ we have

$$
J^{2, b}\left(p^{1, \star}, p^{2, \star}\right) \geq J^{2, b}\left(p^{1, \star}, p^{2}\right) .
$$

The concept is defined analogously for the dynamic Cournot game.

As the players employ Markovian strategies, we define the value functions of the players as a function of their capacities by

$$
\begin{aligned}
& V^{i, b}\left(x_{1}, x_{2}\right)=\sup _{p^{i}} J^{i, b}\left(p^{1}\left(x_{1}, x_{2}\right), p^{2}\left(x_{1}, x_{2}\right)\right), \\
& V^{i, c}\left(x_{1}, x_{2}\right)=\sup _{q^{i}} J^{i, c}\left(q^{1}\left(x_{1}, x_{2}\right), q^{2}\left(x_{1}, x_{2}\right)\right) .
\end{aligned}
$$

Assuming sufficient regularity of the value functions, a sufficient condition for equilibrium can be found by solving the associated system of HJB partial differential equations, see [Dockner et al., 2000, Section 8.2], [Başar and Olsder, 1995, Section 6.5.2]:

$$
\begin{aligned}
& \mathcal{L} V^{i, b}+\sup _{p^{i} \geq 0}\left\{-V_{x_{1}}^{i, b} q_{1}\left(p^{1}, p^{2}\right)-V_{x_{2}}^{i, b} q_{2}\left(p^{1}, p^{2}\right)+p^{i} \cdot q_{i}\left(p^{1}, p^{2}\right)\right\}=r V^{i, b}, \\
& \mathcal{L} V^{i, c}+\sup _{q^{i} \geq 0}\left\{-V_{x_{1}}^{i, c} q^{1}-V_{x_{2}}^{i, c} q^{2}+p_{i}\left(q^{1}, q^{2}\right) \cdot q^{i}\right\}=r V^{i, c},
\end{aligned}
$$

where

$$
\mathcal{L}=\frac{1}{2}\left(\sigma^{1}\right)^{2} \frac{\partial^{2}}{\partial\left(x_{1}\right)^{2}}+\rho \sigma^{1} \sigma^{2} \frac{\partial^{2}}{\partial x_{1} \partial x_{2}}+\frac{1}{2}\left(\sigma^{2}\right)^{2} \frac{\partial^{2}}{\partial\left(x_{2}\right)^{2}}
$$

In order to complete the description of the PDE problem associated with these games we also need to specify boundary conditions. One of these conditions is quite straightforward, we must have $V^{1, \cdot}\left(0, x_{2}\right)=V^{2, \cdot}\left(x_{1}, 0\right)=0$ as there is no profit possible once a firm exhausts their capacity. The other boundary is slightly more complicated. Let $v_{m}^{b}$ be the value function of a monopolist in a Bertrand market, and $v_{m}^{c}$ be the corresponding value function in a Cournot market. We then have the condition $V^{1, \cdot}\left(x_{1}, 0\right)=v_{m}^{\cdot}\left(x_{1}\right)$ and $V^{2, \cdot}\left(0, x_{2}\right)=v_{m}\left(x_{2}\right)$.

We can solve the static Nash equilibrium problems within these equations in both the Bertrand and Cournot case and therefore present the two expanded systems of PDEs. We shall first do this for the Bertrand game and then for the Cournot game.

\subsubsection{Bertrand PDEs}

It is straightforward from (5) and (7) to show the relationship

$$
q_{j}\left(p_{1}, p_{2}\right)=q_{m}\left(p^{j}\right)-\varepsilon q_{i}\left(p_{1}, p_{2}\right), \quad j \neq i .
$$


Therefore, we can rewrite (20) as

$$
\mathcal{L} V^{i, b}-q_{m}\left(p^{j}\right) V_{x_{j}}^{i, b}+\sup _{p^{i} \geq 0}\left\{q_{i}\left(p^{1}, p^{2}\right)\left[p^{i}-\left(V_{x_{i}}^{i, b}-\varepsilon V_{x_{j}}^{i, b}\right)\right]\right\}=r V^{i, b}, \quad i=1,2 ; j \neq i .
$$

Defining the shadow costs

$$
S_{1}^{b} \triangleq V_{x_{1}}^{1, b}-\varepsilon V_{x_{2}}^{1, b} \quad \text { and } \quad S_{2}^{b} \triangleq V_{x_{2}}^{2, b}-\varepsilon V_{x_{1}}^{2, b},
$$

we see that the Nash equilibrium problem implicit in the two PDEs is exactly a static two-player Bertrand type game with marginal costs given by $S_{1}^{b}$ and $S_{2}^{b}$. In other words, we need to solve for a Nash equilibrium of a static game where Firm $i$ sets price $p^{i}$ and obtains profit $q_{i}\left(p^{1}, p^{2}\right) \cdot\left(p^{i}-S_{i}^{b}\right)$.

For linear demand functions, the equilibrium prices are simple to derive assuming that both players are active in equilibrium. In other words, we assume that the asymmetry in cost between the two players is such that both players have positive demand in equilibrium. For $\varepsilon$ small, this will be the case because each firm will be close to having an independent monopoly. We maintain this assumption throughout what follows. The resulting prices, written as functions of the shadow costs, are given by

$$
p^{i, \star}\left(S_{1}^{b}, S_{2}^{b}\right)=\alpha\left(\frac{1-\varepsilon}{2-\varepsilon}\right)+\frac{1}{4-\varepsilon^{2}}\left(2 S_{i}^{b}+\varepsilon S_{j}^{b}\right), \quad j \neq i .
$$

This results in the equilibrium demands, which we also write as a function of the shadow costs:

$$
q_{i}^{\star}\left(S_{1}^{b}, S_{2}^{b}\right)=\frac{\alpha}{\beta(2-\varepsilon)(1+\varepsilon)}-\left(\frac{1}{\beta\left(1-\varepsilon^{2}\right)\left(4-\varepsilon^{2}\right)}\right)\left(\left(2-\varepsilon^{2}\right) S_{i}^{b}-\varepsilon S_{j}^{b}\right), \quad j \neq i .
$$

One can also readily show that the equilibrium profit in the static game is equal to

$$
q_{i}^{\star}\left(S_{1}^{b}, S_{2}^{b}\right) \cdot\left(p^{i, \star}\left(S_{1}^{b}, S_{2}^{b}\right)-S_{i}^{b}\right)=\beta\left(1-\varepsilon^{2}\right)\left(q_{i}^{\star}\left(S_{1}^{b}, S_{2}^{b}\right)\right)^{2} .
$$

We can therefore write $(24)$ as

$$
\mathcal{L} V^{i, b}-q_{m}\left(p^{j, \star}\left(S_{1}^{b}, S_{2}^{b}\right)\right) V_{x_{j}}^{i, b}+\beta\left(1-\varepsilon^{2}\right)\left(q_{i}^{\star}\left(S_{1}^{b}, S_{2}^{b}\right)\right)^{2}=r V^{i, b}, \quad j \neq i,
$$

where explicitly

$$
q_{m}\left(p^{j, \star}\left(S_{1}^{b}, S_{2}^{b}\right)\right)=\frac{\alpha}{\beta(2-\varepsilon)}-\frac{1}{\beta\left(4-\varepsilon^{2}\right)}\left(2 S_{j}^{b}+\varepsilon S_{i}^{b}\right)
$$

\subsubsection{Cournot PDEs}

The analysis of the Cournot system of PDEs is more straightforward as the internal Nash equilibrium problem can be written as a static Cournot game without any additional modification, i.e. we can write $(21)$ as

$$
\mathcal{L} V^{i, c}-q^{j} V_{x_{j}}^{i, c}+\sup _{q^{i} \geq 0}\left\{q^{i}\left[p_{i}\left(q^{1}, q^{2}\right)-V_{x_{i}}^{i, c}\right]\right\}=r V^{i, c}, \quad i=1,2 ; j \neq i .
$$

Hence, defining the shadow costs

$$
S_{1}^{c} \triangleq V_{x_{1}}^{1, c} \quad \text { and } \quad S_{2}^{c} \triangleq V_{x_{2}}^{2, c},
$$


we can see that the static Nash equilibrium problems is exactly a static two-player Cournot type game with marginal costs given by $S_{1}^{c}$ and $S_{2}^{c}$. That is, we need to solve for a Nash equilibrium of a game where Firm $i$ sets $q^{i}$ and obtains profit $q^{i} \cdot\left(p_{i}\left(q^{1}, q^{2}\right)-S_{i}^{c}\right)$.

This calculation is straightforward and results in the equilibrium quantity strategies

$$
q^{i, \star}\left(S_{1}^{c}, S_{2}^{c}\right)=\frac{\alpha}{\beta(2+\varepsilon)}-\frac{1}{\beta\left(4-\varepsilon^{2}\right)}\left(2 S_{i}^{c}-\varepsilon S_{j}^{c}\right)
$$

and the equilibrium profit

$$
q^{i, \star}\left(S_{1}^{c}, S_{2}^{c}\right) \cdot\left(p_{i}\left(q^{1, \star}, q^{2, \star}\right)-S_{i}^{c}\right)=\beta\left(q^{i, \star}\left(S_{1}^{c}, S_{2}^{c}\right)\right)^{2} .
$$

Using these, we arrive at

$$
\mathcal{L} V^{i, c}-q^{j, \star}\left(S_{1}^{c}, S_{2}^{c}\right) V_{x_{j}}^{i, c}+\beta\left(q^{i, \star}\left(S_{1}^{c}, S_{2}^{c}\right)\right)^{2}=r V^{i, c}, \quad i=1,2 ; j \neq i .
$$

\subsubsection{Interpretation and Large Capacity Limits}

We see that (28) and (33) can be seen as equations of the form

$$
\text { Value }=\frac{1}{r}\{\text { Noise }- \text { Competition }+ \text { Profit }\} .
$$

The operator $\mathcal{L}$ is related to the stochastic component of demand coming from the noise term. The second term is denoted "Competition" because it involves a quantity associated with your opponent and the infinitesimal effect on your value function of a change in your opponent's capacity. If your value were independent of your opponents capacity then $V_{x_{j}}^{i, \cdot} \equiv 0$ and this competition term would

not be present. The third term is the equilibrium profit from a static game calculation where the costs are in actuality given by the shadow costs $S_{i}$.

Finally, we note that as $\left(x_{1}, x_{2}\right)$ tends to infinity, we are in a dynamic game of inexhaustible resources, and we expect the value functions to be flat. Hence, all the partial derivatives in (28) and (33) will be zero, and we have

$$
V^{i, c} \rightarrow \frac{1}{r} \beta\left(q^{i, \star}(0,0)\right)^{2}=\frac{\alpha^{2}}{r \beta(2+\varepsilon)^{2}} \quad V^{i, b} \rightarrow \frac{1}{r} \beta\left(q_{i}^{\star}(0,0)\right)^{2}=\frac{\alpha^{2}}{r \beta(2-\varepsilon)^{2}(1+\varepsilon)^{2}} .
$$

These are simply the zero cost static game profit functions discounted at rate $r$ over all time.

\section{Ordinary Differential Game with a Little Competition}

We shall focus our attention on the setting $\sigma^{1}=\sigma^{2} \equiv 0$, i.e. $\mathcal{L} \equiv 0$, and derive an asymptotic approximation for the value functions $V^{i, b}$ and $V^{i, c}$ in powers of $\varepsilon$, the degree of product differentiation. We will then explore the implications of this approximation for equilibrium prices, quantities and economic welfare. Such results are valid for small degrees of product differentiation, that is as a monopoly becomes a duopoly with a little competition: see Figure 1 for a timely example. 


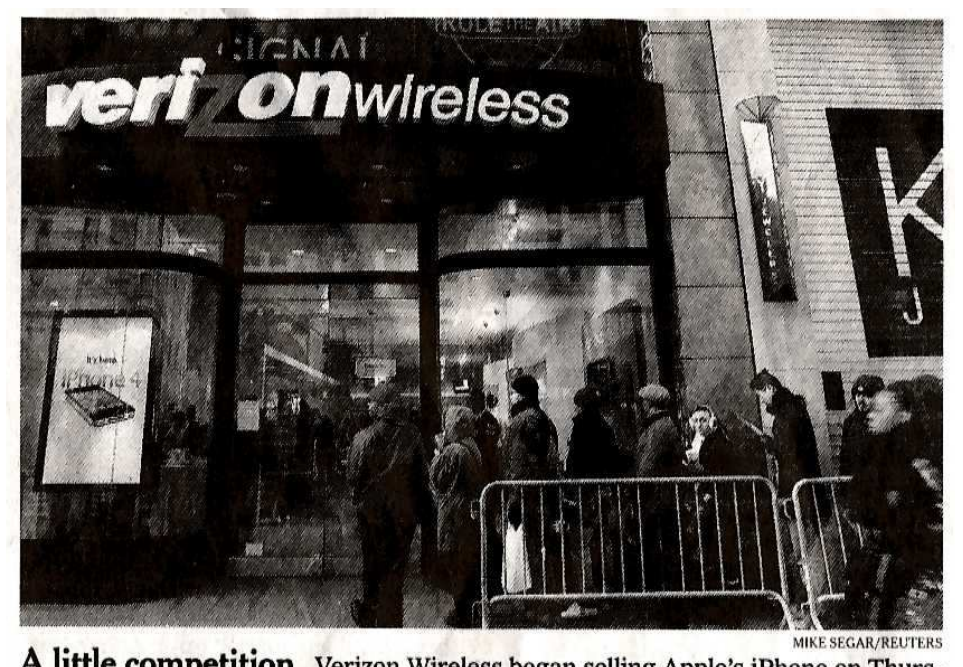

A little competition Verizon Wireless began selling Apple's iPhone on Thursday, ending AT\&T's three-year monopoly on U.S. sales. Above, a store in New York.

Figure 1: From International Herald Tribune, 11 February 2011.

\subsection{Asymptotic Analysis}

The two PDE problems are

$$
\begin{aligned}
& -q_{m}\left(p^{j, \star}\left(S_{1}^{b}, S_{2}^{b}\right)\right) V_{x_{j}}^{i, b}+\beta\left(1-\varepsilon^{2}\right)\left(q_{i}^{\star}\left(S_{1}^{b}, S_{2}^{b}\right)\right)^{2}=r V^{i, b}, \\
& -q^{j, \star}\left(S_{1}^{c}, S_{2}^{c}\right) V_{x_{j}}^{i, c}+\beta\left(q^{i, \star}\left(S_{1}^{c}, S_{2}^{c}\right)\right)^{2}=r V^{i, c}, \quad i=1,2 ; j \neq i .
\end{aligned}
$$

Note that as given above, the $q$ functions in the above PDEs themselves depend on $\varepsilon$. The boundary conditions are that Firm $i$ has zero value when $x_{i}=0$, and Firm $i$ has value function equal to the value function of a monopolist when $x_{i}>0$ and $x_{j}=0$. Moreover as we have already stated, the case $\varepsilon=0$ corresponds to the firms having independent monopolies, so we construct an expansion of the form:

$$
V^{i, \cdot}\left(x_{1}, x_{2}\right)=v_{m}^{\cdot}\left(x_{i}\right)+\varepsilon v_{i}^{(1), \cdot}\left(x_{1}, x_{2}\right)+\varepsilon^{2} v_{i}^{(2), \cdot}\left(x_{1}, x_{2}\right)+\cdots,
$$

where the $\cdot$ stands for $b$ or $c$.

We first expand the functions that arise from the static Bertrand game in (27) and (29):

$$
\begin{aligned}
q_{m}\left(p^{j, \star}\left(s_{1}, s_{2}\right)\right)[\varepsilon] & =q_{m}^{\star}\left(s_{i}\right)+\frac{\varepsilon}{2} q_{m}^{\star}\left(s_{j}\right)+\frac{\varepsilon^{2}}{4} q_{m}^{\star}\left(s_{i}\right)+\cdots, \\
q_{i}^{\star}\left(s_{1}, s_{2}\right)[\varepsilon] & =q_{m}^{\star}\left(s_{i}\right)-\frac{\varepsilon}{2} q_{m}^{\star}\left(s_{j}\right)+\frac{3 \varepsilon^{2}}{4} q_{m}^{\star}\left(s_{i}\right)+\cdots,
\end{aligned}
$$

and that of the Cournot game in (32):

$$
q^{i, \star}\left(s_{1}, s_{2}\right)[\varepsilon]=q_{m}^{\star}\left(s_{i}\right)-\frac{\varepsilon}{2} q_{m}^{\star}\left(s_{j}\right)+\frac{\varepsilon^{2}}{4} q_{m}^{\star}\left(s_{i}\right)+\cdots
$$

where

$$
q_{m}^{\star}(s)=\frac{1}{2 \beta}(\alpha-s) .
$$


Next, we recall the shadow costs for the Bertrand game

$$
S_{i}^{b}=V_{x_{i}}^{i, b}-\varepsilon V_{x_{j}}^{i, b}
$$

and note that as the first term in the expansion (38) for Firm $i$ depends only on the capacity of Firm $i$, we will have that the first term for which the shadow cost is not equal to $V_{x_{i}}^{i, b}$ will be of order $\varepsilon^{2}$. Hence, up to order $\varepsilon$ we have that $S_{b}^{i}=V_{x_{i}}^{i, b}$. For the second-order term however, we will need to consider the additional effect of this cross-partial derivative within the shadow cost. This is a straightforward, albeit lengthy, calculation which we omit.

Before deriving the PDE problems for the first and second-order terms in the approximation, we can use (39)-(41) to understand what type of behavior we expect. Foremost, we notice that the zero-order terms are identical across the three terms. Therefore, we expect that the monopoly value functions will be the same. The next thing to notice is that the functions that enter into the profit term, (41) in Cournot and (40) in Bertrand, are identical up to order $\varepsilon$. Moreover, based on the expansion, $V_{x_{j}}^{i,}$ will be of order $\varepsilon$ and therefore the only term entering into the competition term from this quantity functions are the zero-order terms of (41) for Cournot and (39) for Bertrand. In addition, the constant in the profit term is identical at order $\varepsilon$ and, as we have said, the shadow costs are also identical at order $\varepsilon$. Therefore, we expect the first-order approximation functions to be the same across the two game types. This is a surprising fact which we shall return to. Finally, at order $\varepsilon^{2}$, we see several places where terms are not equal. Therefore, we have no reason to suspect a priori that the second-order approximation functions would be the same.

\subsubsection{Zero-order Expansion: Monopoly}

Carrying out the asymptotic expansion, we find that the zeroth-order PDE problem for both games and both players reduces to solving

$$
\frac{1}{4 \beta}\left(\alpha-v_{m}^{\prime}\right)^{2}-r v_{m}=0
$$

with $v_{m}(0)=0$ and $\lim _{x \rightarrow \infty} v_{m}^{\prime}(x)=0$. In other words, given the solution to this ODE we have

$$
v_{m}^{c}\left(x_{i}\right) \equiv v_{m}^{b}\left(x_{i}\right) \equiv v_{m}\left(x_{i}\right) .
$$

Proposition 4.1.1 (Prop. 3.1 in [Ledvina and Sircar, 2011]). The solution to (44) is given by

$$
v_{m}(x)=\frac{\alpha^{2}}{4 \beta r}\left[\mathbf{W}\left(-e^{-\mu x-1}\right)+1\right]^{2},
$$

where $\mu=(2 \beta r) / \alpha$ and $\mathbf{W}$ is the Lambert $\mathbf{W}$ function defined by the relation $Y=\mathbf{W}(Y) e^{\mathbf{W}(Y)}$ with domain $Y \geq-e^{-1}$.

Proof. It is straightforward to check that the Lambert $\mathbf{W}$ function satisfies $\mathbf{W}(z)<0$ for $z \in$ $\left[-e^{-1}, 0\right), \mathbf{W}\left(-e^{-1}\right)=-1, \mathbf{W}(0)=0$, and $\mathbf{W}^{\prime}(z)=\mathbf{W}(z) /(z(1+\mathbf{W}(z)))$ for $z>-e^{-1}$, and therefore that Eqn. (45) indeed satisfies Eqn. (44) and the boundary condition $v_{M}(0)=0$. We note that the restriction of the domain of $\mathbf{W}$ to $\left[-e^{-1}, \infty\right)$ is sufficient as the argument to $\mathbf{W}$ in Eqn. (45) is equal to $-e^{-1}$ when $x=0$ and increases to zero as $x$ increases to infinity.

We therefore have an explicit solution, up to a special function, of the monopoly problem. We plot this function in Figure 2. 


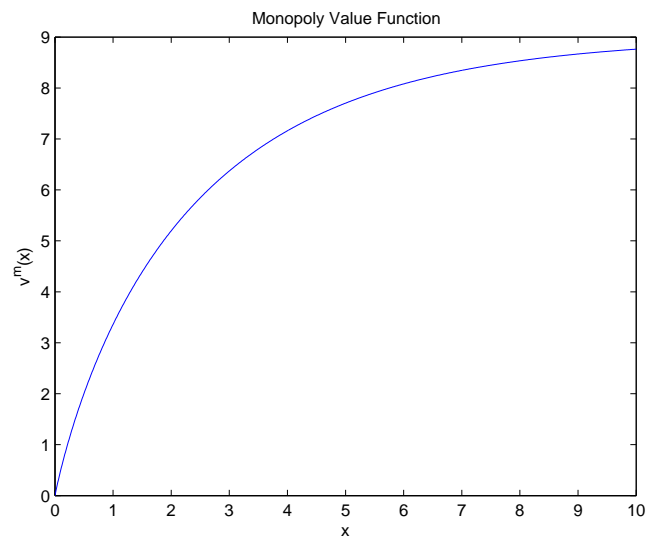

Figure 2: Plot of Monopoly Value Function

\subsubsection{First-order Expansion}

We define

$$
\lambda(x) \triangleq q_{m}^{\star}\left(v_{m}^{\prime}(x)\right)=\frac{1}{2 \beta}\left(\alpha-v_{m}^{\prime}(x)\right),
$$

and the differential operator

$$
\mathcal{A} \triangleq \lambda\left(x_{1}\right) \frac{\partial}{\partial x_{1}}+\lambda\left(x_{2}\right) \frac{\partial}{\partial x_{2}}+r .
$$

It turns out that the approximation of the system of PDEs at order $\varepsilon$ for both Cournot and Bertrand games are the same, and reduces to solving

$$
\mathcal{A} v_{i}^{(1)}=-\beta \lambda\left(x_{1}\right) \lambda\left(x_{2}\right),
$$

with $v_{i}^{(1)}\left(0, x_{2}\right)=v_{i}^{(1)}\left(x_{1}, 0\right)=0$ for $i=1,2$. Before doing anything we see immediately by the symmetry of the operator and the symmetry of the right-hand-side that the solution $v_{i}^{(1)}$ is symmetric in $x_{1}$ and $x_{2}$ and therefore $v^{(1)}\left(x_{1}, x_{2}\right):=v_{1}^{(1)}\left(x_{1}, x_{2}\right) \equiv v_{2}^{(1)}\left(x_{1}, x_{2}\right)$.

Proposition 4.1.2. The solution of (48) is given, for $x_{1}>x_{2}$ by

$$
v^{(1)}\left(x_{1}, x_{2}\right)=\frac{\alpha^{2}}{4 \beta r}\left(e^{-r \Lambda\left(x_{2}\right)}\left(1+r \Lambda\left(x_{2}\right)\right)-e^{-r \Lambda\left(x_{1}\right)}\left(1-r \Lambda\left(x_{2}\right)\right)+e^{-r\left(\Lambda\left(x_{1}\right)+\Lambda\left(x_{2}\right)\right)}-1\right),
$$

where

$$
\Lambda(x) \triangleq \int_{0}^{x} \frac{1}{\lambda(u)} d u=-\frac{1}{r} \log \left(-\mathbf{W}\left(-e^{-\mu x-1}\right)\right),
$$

and, for $x_{2} \geq x_{1}$, by reversing the roles of $x_{1}$ and $x_{2}$ in (49). Moreover, for $i=1,2$ we have

$$
v_{i}^{(1), b}\left(x_{1}, x_{2}\right) \equiv v_{i}^{(1), c}\left(x_{1}, x_{2}\right) \equiv v^{(1)}\left(x_{1}, x_{2}\right) .
$$

Proof. See the proof of Proposition 3.2 in [Ledvina and Sircar, 2011]. 
Up to order $\varepsilon$ we have found no difference between Cournot and Bertrand. Moreover, the approximation to the value function is given analytically. We plot this function in Figure 3(a) and the corresponding value function surface up to order $\varepsilon$ with $\varepsilon=0.2$ in Figure 3(b). The first order correction is negative over the entire domain which matches intuition as this is the correction due to a small amount of competition to the value of a monopolist. Moreover, this correction is larger when the total capacity $x_{1}+x_{2}$ is larger.

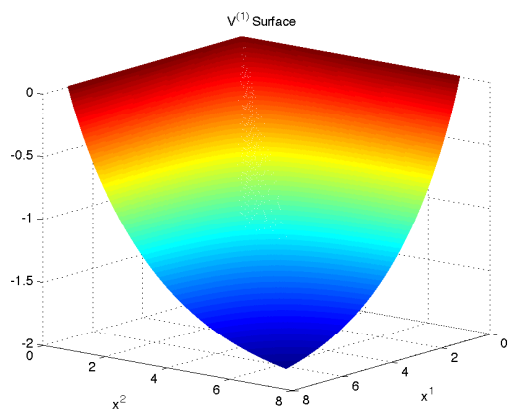

(a) $v^{(1)}$ : Surface

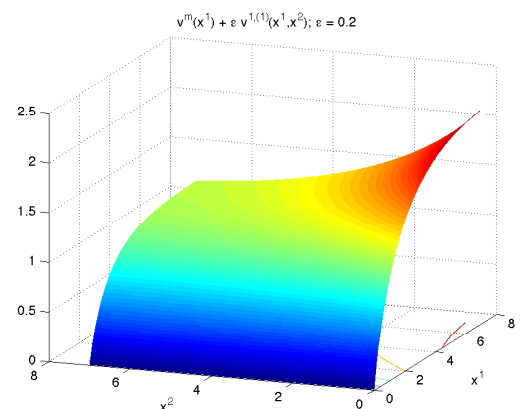

(b) $v_{m}\left(x_{1}\right)+\varepsilon v^{(1)}\left(x_{1}, x_{2}\right)$ : Surface

Figure 3: $v^{(1)}$ and $v_{m}\left(x_{1}\right)+\varepsilon v^{(1)}\left(x_{1}, x_{2}\right)$. First-order correction function and full value function approximation up to $O(\varepsilon)$ for $\varepsilon=0.2$.

\subsubsection{Second-order Expansion}

We now turn to the approximation at order $\varepsilon^{2}$. Using the expansions in $\varepsilon$ for the two PDEs, we find the following PDE problems

$$
\begin{aligned}
\mathcal{A} v_{i}^{(2), b}= & \frac{1}{2} \lambda\left(x_{i}\right) \frac{\partial v^{(1)}}{\partial x_{j}}+\frac{1}{2}\left(\lambda\left(x_{i}\right) \frac{\partial v^{(1)}}{\partial x_{j}}+\lambda\left(x_{j}\right) \frac{\partial v^{(1)}}{\partial x_{i}}\right) \\
& +\frac{1}{4} \lambda\left(x_{j}\right)^{2}-\lambda\left(x_{i}\right)^{2}+\frac{1}{2} \frac{\partial v^{(1)}}{\partial x_{j}} \frac{\partial v^{(1)}}{\partial x_{j}}+\frac{1}{4}\left(\frac{\partial v^{(1)}}{\partial x_{i}}\right)^{2}, \\
\mathcal{A} v_{i}^{(2), c}= & \frac{1}{2} \lambda\left(x_{i}\right) \frac{\partial v^{(1)}}{\partial x_{j}}-\frac{1}{2}\left(\lambda\left(x_{i}\right) \frac{\partial v^{(1)}}{\partial x_{j}}+\lambda\left(x_{j}\right) \frac{\partial v^{(1)}}{\partial x_{i}}\right) \\
& +\frac{1}{4} \lambda\left(x_{j}\right)^{2}+\frac{1}{2} \lambda\left(x_{i}\right)^{2}+\frac{1}{2} \frac{\partial v^{(1)}}{\partial x_{j}} \frac{\partial v^{(1)}}{\partial x_{j}}+\frac{1}{4}\left(\frac{\partial v^{(1)}}{\partial x_{i}}\right)^{2},
\end{aligned}
$$

with $v_{i}^{(2), \cdot}\left(x_{1}, 0\right)=v_{i}^{(2), \cdot}\left(0, x_{2}\right)=0$ for $i=1,2$ for both Bertrand and Cournot. The elements that are boxed are the only differences between the two PDE problems.

Proposition 4.1.3. There exists an analytic solution to (51) which is presented in Appendix A.

Proposition 4.1.4. There exists an analytic solution to (52) which is presented in Appendix B.

We plot the solution that results from Proposition 4.1.3 in Figure 4 (a) and that from Proposition 4.1.4 in Figure 4 (b). 


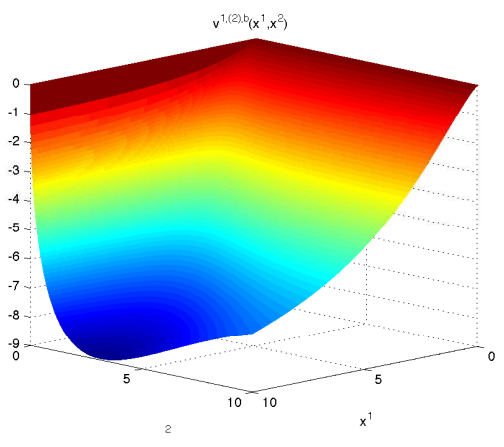

(a) $v_{1}^{(2), b}:$ Surface

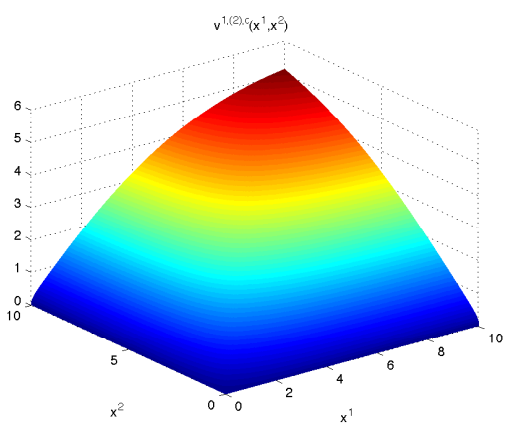

(b) $v_{1}^{(2), c}$ : Surface

Figure 4: Second-order correction surfaces

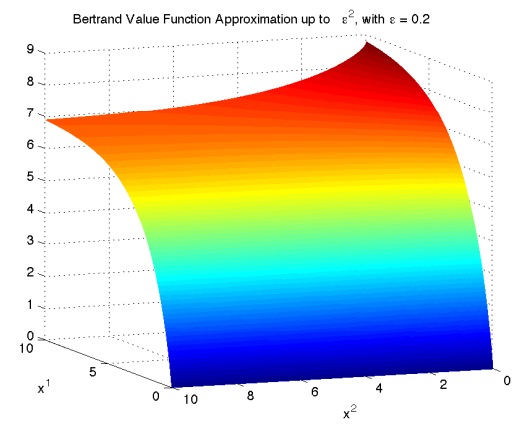

(a) Bertrand

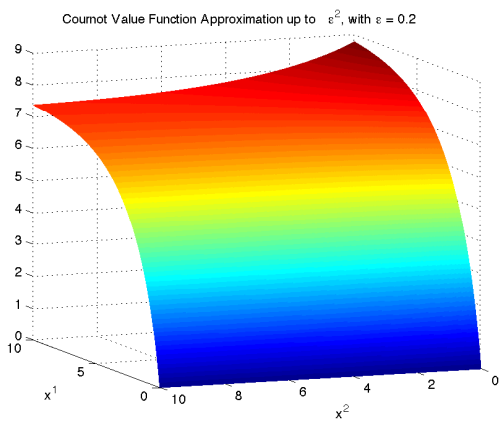

(b) Cournot

Figure 5: Plots of $v_{m}\left(x_{1}\right)+\varepsilon v^{(1)}\left(x_{1}, x_{2}\right)+\varepsilon^{2} v_{1}^{(2) \cdot}\left(x_{1}, x_{2}\right)$ with $\varepsilon=0.2$

Our first observation is that the second-order correction in the Bertrand game is everywhere negative. Therefore, an increased amount of competition, as measured by $\varepsilon$, is detrimental to the value of a firm in a Bertrand game.

The second observation is that the Cournot second-order correction is everywhere positive. Therefore, the value of a firm in a Cournot market is higher than in a Bertrand market when one takes into account these second-order effects, or in other words, the correction of order $\varepsilon^{2}$ has the opposite effect on a firm in a Cournot market as compared to a firm in a Bertrand market. This leads to the conclusion that the value of a firm is greater in a Cournot market than in a Bertrand market.

We see just this effect in Figure 5. In this figure where $\varepsilon=0.2$, we see that the our approximation to the value function in the Cournot game is greater than the value function in the Bertrand game. The monopoly boundary is identical and the zero boundary is identical, and moreover in both cases, the value of a firm decreases with respect to the capacity of one's rival. However, in a Bertrand game relative to a Cournot game, the decrease is at a faster rate. This leads to the lower value in a Bertrand game when both firms have a high level of a capacity. As capacities increase indefinitely, the game approaches something of a repeated static game as the changes in capacity are small relative to the overall size of capacity and therefore do not affect the strategies of firms to an appreciable amount. Therefore, the lower value in a Bertrand market when both firms have a large capacity is similar to the fact that the static Bertrand game is more competitive than the static Cournot game, and this becomes exact in the limit as $x_{1}, x_{2} \rightarrow \infty$, which can be seen in (35). 


\subsection{Prices, quantities}

We have laid the groundwork for our comparison of Bertrand and Cournot competition in the limit of small product differentiation. As the monopoly value functions are identical and the first-order value functions are identical, we have that in markets with very little competition, the type of market is immaterial up to order $\varepsilon$ on any measure of consumer well-begin.

Given the value functions approximated up to order $\varepsilon^{2}$, which are now different for Cournot and Bertrand, these can be used to approximate price and demand, which in turn are used as inputs to drive the system of ODEs describing the capacity dynamics with $\sigma^{i} \equiv 0$. The resulting capacity trajectories for Firm 1 in the two game types are displayed in Figure 6. We observe that the game ends sooner in the Bertrand market. This again aligns with previous intuition that a Bertrand market is more competitive than a Cournot market. This increased level of competition leads to a faster rate of capacity depletion, ceteris paribus.

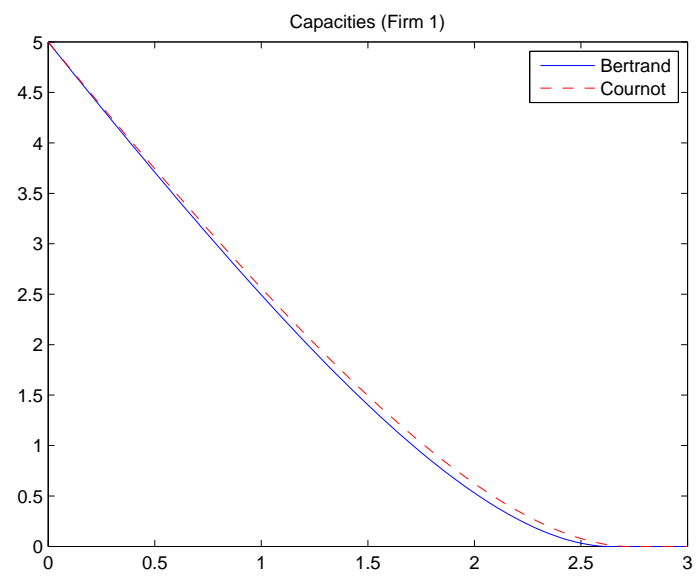

Figure 6: Deterministic Capacity Paths with $\varepsilon=0.2$

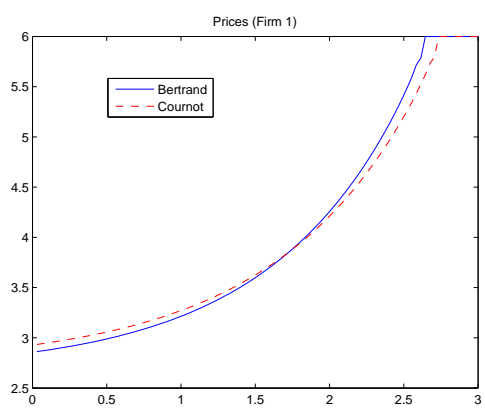

(a) Price

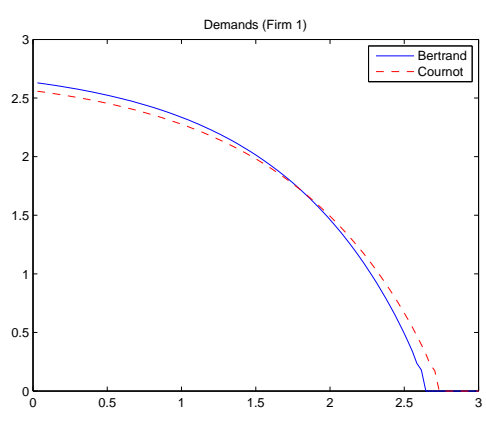

(b) Demand

Figure 7: Deterministic Price and Demand Paths with $\varepsilon=0.2$

However, this intuition that comes from the static game breaks down when we observe the resulting price paths in Figure 7 (a), and the corresponding paths of demand in Figure 7 (b). The static game 
intuition says that Bertrand should have lower prices and higher quantities. This is true at the beginning of the game when both firms have a large capacity. However, as the firms deplete their capacities, we see that the price in the Bertrand market increases until it finishes above that in the Cournot market. Likewise, the quantity begins higher in the Bertrand market, but eventually drops below the quantity in the Cournot market. The dynamic nature of the game and the dependency on firms' capacities leads to these counterintuitive results.

\section{Stochastic Differential Game with Greater Competition}

We now turn our focus to the stochastic differential game, i.e. where $\sigma^{1}>0$ and $\sigma^{2}>0$. This will allow us to study larger degrees of product differentiation than for which the asymptotic approximation is reasonable to use. However, we still cannot consider the limit as $\varepsilon$ approaches 1, because $\varepsilon=1$ corresponds to homogeneous goods, which results in the well-known Bertrand paradox: only the firm with the lowest marginal cost receives any demand, and if all firms have equal cost then equilibrium prices are equal to marginal cost and all firms earn zero profits. As partial derivatives of the value function take the place of marginal costs, i.e. they represent shadow costs due to scarcity, the implementation of the Bertrand differential game with homogeneous goods is particularly unstable due to highly discontinuous behavior in the payoff functions. This can be overcome in the static setting but is a difficult problem in the differential game.

\subsection{Numerical Analysis}

For the ordinary differential game, we do obtained in Section 4 an analytical approximation to these functions which provide a great deal of insight into these games. On the other hand, numerical solution of systems of nonlinear first-order PDEs using finite-difference methods is difficult without an appropriate regularizing method, such as approaching it in the limit of stochastic games as the randomness goes to zero.

Contrarily, the stochastic differential game is well suited to a solution using the method of finitedifferences. This is due to the smoothing effect that the second-order operator provides to the solution. For example, one can prove somewhat general existence results for systems of PDEs of this type provided the second-order term is uniformly elliptic and the domain is smooth and bounded, see [Bensoussan and Frehse, 2002, Chapter 3]. In our case, the ellipticity requirement is satisfied with $\sigma^{1}>0$ and $\sigma^{2}>0$. Our natural domain, however, is not smooth and hence such a result cannot be applied directly.

Repeating the small $\varepsilon$ expansion in the stochastic game does not yield an analytic solution even in the zeroth-order monopoly term. Throughout what follows, unless otherwise noted, we fixed the parameter values $\sigma^{1}=0.6, \sigma^{2}=0.6, \rho=0.1, \alpha=6, \beta=1$ and $\varepsilon=0.4$. We present in Figure 8 the numerical approximations to the value functions in the Bertrand (a) and Cournot (b) games.

Although possibly difficult to see in these plots, the value in the Cournot game is greater than the value in the Bertrand game when both firms have large levels of capacity. Moreover, the axis values are identical for the two game types. However, unlike in the deterministic approximation, the Bertrand value function is actually greater than the Cournot value function when one's rival 


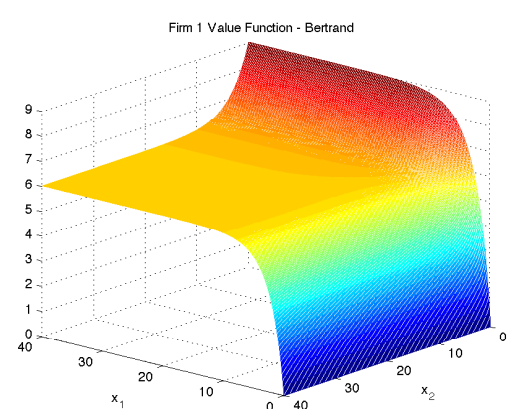

(a) Bertrand

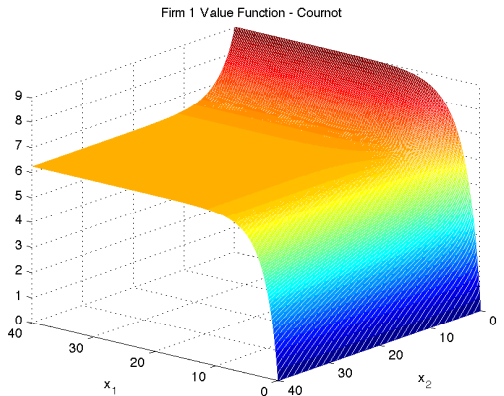

(b) Cournot

Figure 8: Value Function Surfaces in Stochastic Game with $\varepsilon=0.4$

has very low levels of capacity. This is not readily observable from these figures but can be seen by examining the surface one obtains from taking the difference between the Cournot and Bertrand value functions. Therefore, for large enough values of $\varepsilon$ there are effects in the stochastic game which cannot be captured using the second-order approximation that is relevant to the deterministic game.

\subsection{Prices, quantities and welfare}

In the deterministic game, we could use our computed price and quantity strategies to numerically solve the ODEs for capacity and thereby obtain for any fixed initial capacities, the trajectories of capacity, price and demand. However, in the stochastic game, we cannot simply solve for the trajectories as each one depends on the realization of the path of our Brownian motions. We can simulate the Brownian paths and thereby simulate these paths, but the results are then dependent on the particular realization of the randomness. In order to compare across market types while controlling for the randomness, we fix a realization of our two-dimensional Brownian motion and then use this to simulate the desired values in the two markets. A prototypical example of the path of capacity of Firm 1 in the two games is presented in Figure 9.

We see that the time of the Bertrand game is shorter than the Cournot game. This is exactly what we would expect from the results of the deterministic approximation. Hence, one would expect the prices and demands to have similar features to those in the deterministic game. Figure 10 (a) is the corresponding price paths and Figure 10 (b) is the demand paths. Comparing these figures to those in the deterministic game, we see exactly the same qualitative behavior. The prices in the Bertrand market are initially lower, but as firms exhaust their capacities, these prices increase to a level above those in the Cournot market. Similarly, the demands in the Bertrand market are higher initially and lower by the end of the game. Hence, the deterministic approximation captures the qualitative features of the paths of capacity, price and demand quite well.

For given levels of capacity $x_{1}$ and $x_{2}$, we define $\theta \triangleq \tan ^{-1}\left(x_{2} / x_{1}\right)$. This is the angle in $\left(x_{1}, x_{2}\right)$ space that corresponds to the given capacity levels. Therefore, if we fix the total market size $x_{1}+x_{2}$, we can use $\theta \in[0, \pi / 2]$ to measure the variation in price and demand as a function of market share. We plot in Figure 11 the demand of Firm 1 in the two market types. In Figure 11 (a) we have $\varepsilon=0.2$ and in 11 (b) we double the size of $\varepsilon$ to 0.4 . Similarly, we plot the prices of Firm 1 in the 


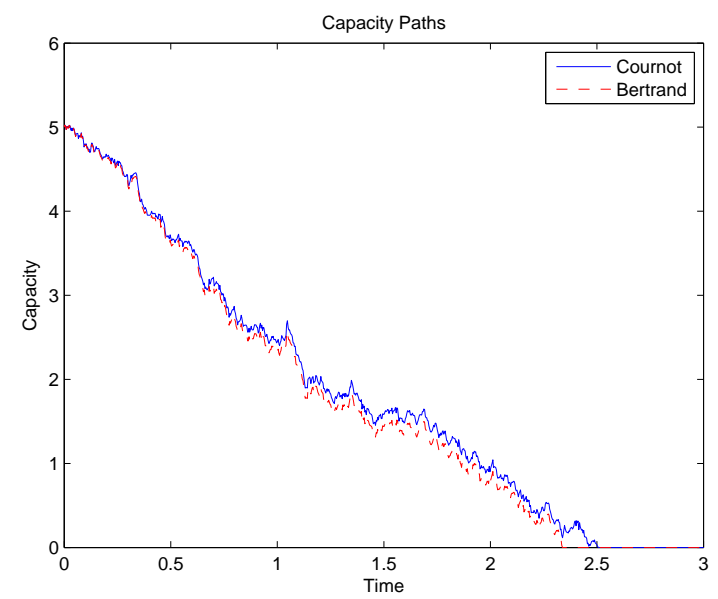

Figure 9: Stochastic Capacity Path Realization with $\varepsilon=0.4$

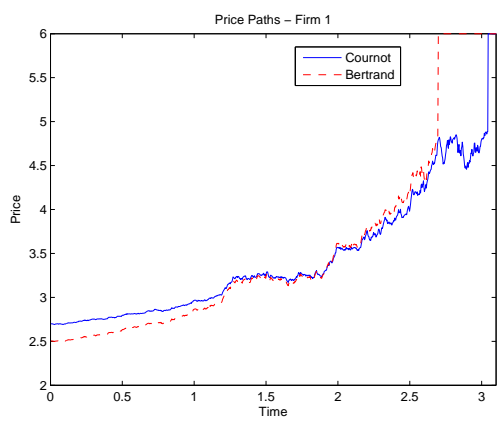

(a) Price

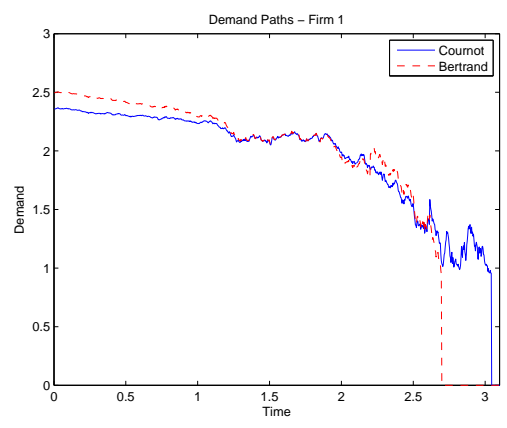

(b) Demand

Figure 10: Stochastic Price and Demand Paths with $\varepsilon=0.4$

two market types as a function of market share in Figure 12, where again $\varepsilon=0.2$ in (a) and $\varepsilon=0.4$ in (b).

Our first observation is that the static game intuition that Bertrand has lower prices and higher quantities does hold in these numeric solutions. This is not a contradiction with previous statements. In the previous sections where we stated that prices and quantities did not obey this relationship, we were describing these values on a path over time. The primary driving force for the results there was the fact that the Bertrand game ended sooner and therefore prices were driven up near the end of the game. In contrast, here we are looking at the equilibrium strategies only as a function of $\left(x_{1}, x_{2}\right)$ fixed, not varying in time.

Furthermore, the spread between the Bertrand and Cournot values, be it price or quantity, is increasing with $\varepsilon$. Thus, in a market with highly substitutable goods, the differences between price competition and quantity competition are magnified. This is a natural statement as the limiting case, $\varepsilon=1$ which corresponds to homogeneous goods, results in a very wide disparity between Cournot and Bertrand competition.

The left-hand-side of these plots, where $\theta=0$, corresponds to Firm 1 having total market share. 


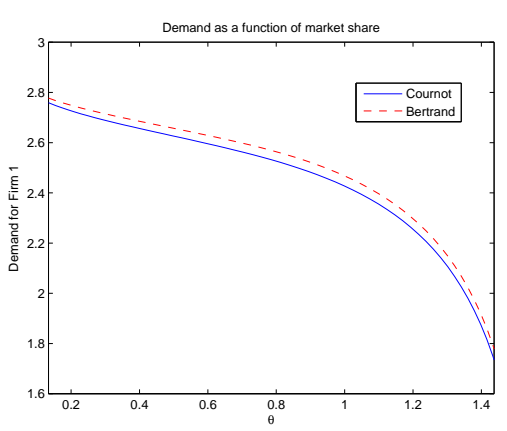

(a) $\varepsilon=0.2$

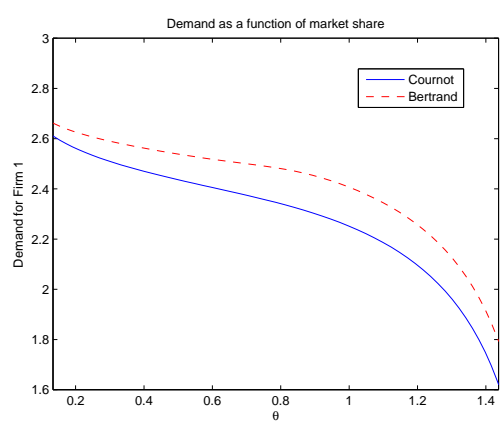

(b) $\varepsilon=0.4$

Figure 11: Demand as a function of market share

Here we see that Firm 1 sets the lowest prices and the highest quantities. The right-hand-side is the opposite situation, Firm 1 has no market share and thus sets the highest prices and lowest quantities. There is a minimum in price near the equal market share level for $\varepsilon$ large enough. Otherwise, the lowest price can be obtained from the one firm with the highest market share. However, note that the average price is lowest when both firms have equal market share, regardless of the value of $\varepsilon$. Similarly, the highest average quantity is obtained when market share is equal across the two firms. Therefore, this model predicts that consumers are better off when firms have roughly equal levels of capacity, or in another sense, firms are roughly of the same size.

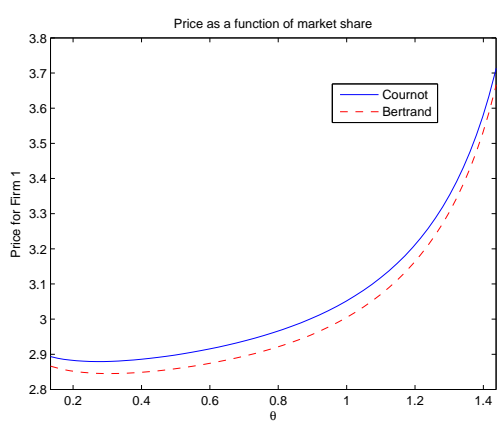

(a) $\varepsilon=0.2$

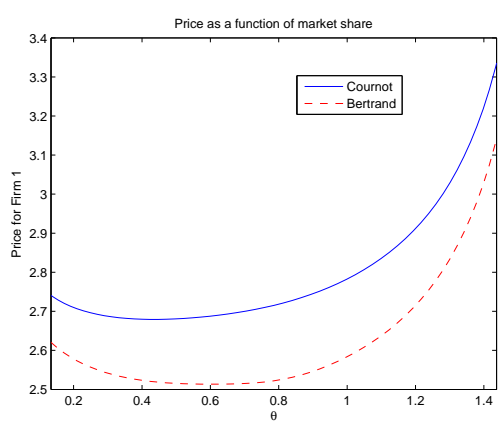

(b) $\varepsilon=0.4$

Figure 12: Price as a function of market share

The previous discussion is somewhat loose in that low average prices may or may not make consumers better off. Economic welfare, as we have already defined, is one measure often used in economics to quantify just this notion. That is, a market with higher economic welfare is better for the economy in some sense.

We plot the difference $E W^{c}-E W^{b}$ in Figure 13. Our first observation is that for almost all levels of capacity, economic welfare is greater in a Bertrand market relative to a Cournot market. However, when both firms have sufficiently exhausted their respective capacities, this relationship reverses and we see that economic welfare is higher in the Cournot market. Hence, for large similarly sized firms with a long time until exhaustion of capacity, the economy is better off if they compete by setting prices. However, near the origin where both are near to exhaustion, the economy would be better off if they were to compete in quantities. 


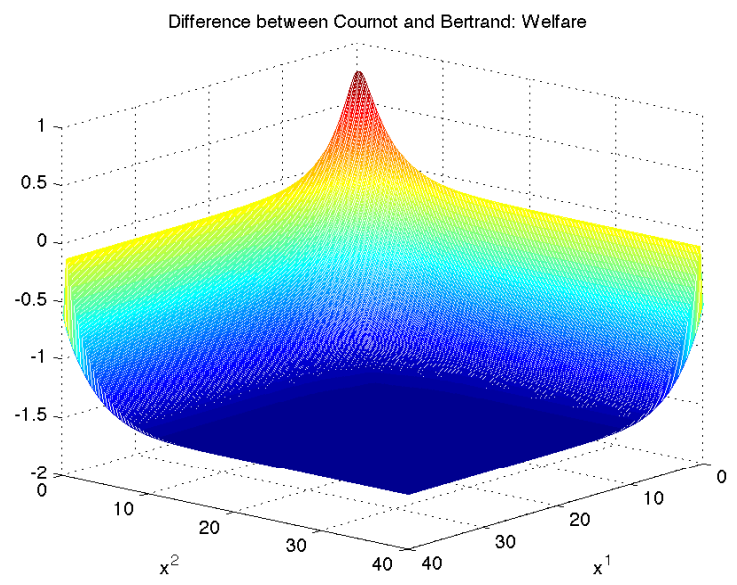

Figure 13: Welfare Difference between dynamic Cournot and Bertrand with $\varepsilon=0.4$

\section{A Second-Order Approximation: Bertrand}

We give the solution of the PDEs Eqn. (51) for $v_{i}^{(2), b}$. Using the solution to $v_{i}^{(1), b}$ found in Proposition 4.1.2, and making the same change of variables as used in the proof of that Proposition, namely $(\xi, \eta)=\left(\Lambda\left(x_{1}\right), \Lambda\left(x_{2}\right)\right)$, and solving, leads to

$$
\begin{aligned}
v_{1}^{(2), b}\left(x_{1}, x_{2}\right)= & \frac{\alpha^{2}}{8 \beta} e^{-r \Lambda\left(x_{2}\right)}\left[-\frac{\Lambda\left(x_{2}\right)^{2}}{2}\left(\frac{2 r e^{r \Lambda\left(x_{2}\right)}}{e^{r \Lambda\left(x_{2}\right)}-1}+\frac{r \varphi_{1}}{1-e^{-r \Lambda\left(x_{1}\right)}}\right)-\frac{\Lambda\left(x_{2}\right)\left(1-\varphi_{1}\right)}{1-e^{-r \Lambda\left(x_{1}\right)}}\right. \\
& +\frac{3}{2 r}\left(e^{r \Lambda\left(x_{2}\right)}-1\right)-\frac{\left(1-\varphi_{1}\right)^{2}}{2 r\left(e^{r \Lambda\left(x_{2}\right)}\left(1-e^{-r \Lambda\left(x_{1}\right)}\right)\right)}+\frac{1-\varphi_{1}}{2 r} \\
& \left.-\frac{3}{r}\left(e^{r \Lambda\left(x_{2}\right)}-\varphi_{1}^{2} e^{-r \Lambda\left(x_{2}\right)}-1+\varphi_{1}^{2}-2 \varphi_{1} r \Lambda\left(x_{2}\right)\right)\right],
\end{aligned}
$$

for $x_{1}>x_{2}$ where $\varphi_{1}=\exp \left\{-r\left(\Lambda\left(x_{1}\right)-\Lambda\left(x_{2}\right)\right)\right\}$. For $x_{2} \geq x_{1}$,

$$
\begin{aligned}
v_{1}^{(2), b}\left(x_{1}, x_{2}\right)= & \frac{\alpha^{2}}{8 \beta} e^{-r \Lambda\left(x_{1}\right)}\left[-\frac{\Lambda\left(x_{1}\right)^{2}}{2}\left(\frac{r e^{r \Lambda\left(x_{1}\right)}}{e^{r \Lambda\left(x_{1}\right)}-1}+\frac{2 r \varphi_{2}}{1-e^{-r \Lambda\left(x_{2}\right)}}\right)-\frac{2 \Lambda\left(x_{1}\right)\left(1-\varphi_{2}\right)}{1-e^{-r \Lambda\left(x_{2}\right)}}\right. \\
& +\frac{3}{2 r}\left(e^{r \Lambda\left(x_{1}\right)}-1\right)-\frac{\left(1-\varphi_{2}\right)^{2}}{r e^{r \Lambda\left(x_{1}\right)}\left(1-e^{-r \Lambda\left(x_{2}\right)}\right)}+\frac{1-\varphi_{2}}{r} \\
& \left.-\frac{3}{r}\left(e^{r \Lambda\left(x_{1}\right)}-2 r \Lambda\left(x_{1}\right)-e^{-r \Lambda\left(x_{1}\right)}\right)\right]
\end{aligned}
$$

where $\varphi_{2}=\exp \left\{-r\left(\Lambda\left(x_{2}\right)-\Lambda\left(x_{1}\right)\right)\right\}$. We omit the details of this lengthy calculation. 


\section{B Second-Order Approximation: Cournot}

We first define the functions $\bar{v}_{i}^{(2)} \triangleq v_{i}^{(2), b}-v_{i}^{(2), c}$ for $i=1,2$. Linearity of $\mathcal{A}$ and the two PDEs, (51) and (52), imply

$$
\mathcal{A} \bar{v}_{i}^{(2)}=\lambda\left(x_{i}\right) \frac{\partial v^{(1)}}{\partial x_{j}}+\lambda\left(x_{j}\right) \frac{\partial v^{(1)}}{\partial x_{i}}-\frac{3}{2} \beta \lambda\left(x_{i}\right)^{2},
$$

with zero boundary conditions.

Proposition B.0.1. The solution of (55) is given, for $i=1$ and $x_{1}>x_{2}$ by

$$
\begin{aligned}
\bar{v}_{1}^{(2)}\left(x_{1}, x_{2}\right) & =e^{-r \Lambda\left(x_{2}\right)}\left[G\left(\Lambda\left(x_{2}\right), \varphi_{1}\right)\right. \\
& \left.-\frac{3 \alpha^{2}}{8 \beta r}\left\{e^{r \Lambda\left(x_{2}\right)}-1-\varphi_{1}^{2}\left(e^{-r \Lambda\left(x_{2}\right)}-1\right)-2 r \varphi_{1} \Lambda\left(x_{2}\right)\right\}\right],
\end{aligned}
$$

and for $x_{2} \geq x_{1}$ by

$$
\begin{aligned}
\bar{v}_{1}^{(2)}\left(x_{1}, x_{2}\right) & =e^{-r \Lambda\left(x_{1}\right)}\left[G\left(\Lambda\left(x_{1}\right), \varphi_{2}\right)\right. \\
& \left.-\frac{3 \alpha^{2}}{8 \beta r}\left\{e^{r \Lambda\left(x_{1}\right)}-e^{-r \Lambda\left(x_{1}\right)}-2 r \Lambda\left(x_{1}\right)\right\}\right],
\end{aligned}
$$

where $\varphi_{i}=\exp \left\{-r\left(\Lambda\left(x_{i}\right)-\Lambda\left(x_{j}\right)\right)\right\}, i=1,2$ and $j \neq i$, and where

$$
\begin{aligned}
G(y, k)= & \frac{1+k^{2}}{r}\left(e^{-r y}-1\right) \\
& +y\left[2-\frac{1}{k}+k\left(1+\log \left(1-e^{-r y}\right)\right)\right. \\
& \left.-\log \left(1-e^{r y}\right)-k \log \left(1-\frac{e^{r y}}{k}\right)+\log \left(1-k e^{-r y}\right)\right] \\
& -\left(\frac{2}{r}-\frac{1}{r k}-\frac{k}{r}\right)\left(\log \left(k-e^{r y}\right)-\log (k-1)\right) \\
& -\frac{1}{r}\left(k L i_{2}\left(e^{-r y}\right)+L i_{2}\left(e^{r y}\right)+k L i_{2}\left(\frac{e^{r y}}{k}\right)+L i_{2}\left(k e^{-r y}\right)\right) \\
& +\frac{1}{r}\left((k+1) L i_{2}(1)+k L i_{2}\left(\frac{1}{k}\right)+L i_{2}(k)\right) .
\end{aligned}
$$

For $i=2$, we find by symmetry that $\bar{v}_{2}^{(2)}\left(x_{1}, x_{2}\right)=\bar{v}_{1}^{(2)}\left(x_{2}, x_{1}\right)$. The necessary details of the Li $i_{2}$ function are given in Remark B.1.

Remark B.1 (Polylogarithm). The polylogarithm function $L i_{n}$ is defined in general by the power series

$$
L i_{n}(z)=\sum_{k=1}^{\infty} \frac{z^{k}}{k^{n}} .
$$

This representation, however, is not of much use here. Instead, we require the alternative representation, from which the function obtains its name, namely

$$
L i_{n+1}(z)=\int_{0}^{z} \frac{L i_{n}(t)}{t} d t
$$


with the base case being given by the logarithm function, i.e. $L i_{1}(z)=-\log (1-z)$. Therefore,

$$
\operatorname{Li}_{2}(z)=-\int_{0}^{z} \frac{\log (1-t)}{t} d t
$$

Proof of Prop B.0.1. The first step, again, is to make the change of variables

$$
(\xi, \eta)=\left(\Lambda\left(x_{1}\right), \Lambda\left(x_{2}\right)\right), \text { and } w^{i}(\xi, \eta)=e^{\frac{r}{2}(\xi+\eta)} \bar{v}_{i}^{(2)}\left(\Lambda^{-1}(\xi), \Lambda^{-1}(\eta)\right),
$$

which results in the PDE problems

$$
\begin{aligned}
& \frac{\partial w^{1}}{\partial \xi}+\frac{\partial w^{1}}{\partial \eta}=e^{\frac{r}{2}(\xi+\eta)}\left(-\frac{3 \alpha^{2}}{8 \beta}\left(1-e^{-r \xi}\right)^{2}+g(\xi, \eta)\right), \\
& \frac{\partial w^{2}}{\partial \xi}+\frac{\partial w^{2}}{\partial \eta}=e^{\frac{r}{2}(\xi+\eta)}\left(-\frac{3 \alpha^{2}}{8 \beta}\left(1-e^{-r \eta}\right)^{2}+g(\xi, \eta)\right),
\end{aligned}
$$

with $w^{i}(\xi, 0)=w^{i}(0, \eta)=0$ for all $\xi, \eta \geq 0$ and where

$$
\begin{aligned}
g(\xi, \eta)= & \frac{\alpha}{2 \beta}\left(1-e^{-r \xi}\right) \frac{\partial v^{(1)}}{\partial x^{2}}\left(\Lambda^{-1}(\xi), \Lambda^{-1}(\eta)\right) \\
& +\frac{\alpha}{2 \beta}\left(1-e^{-r \eta}\right) \frac{\partial v^{(1)}}{\partial x^{1}}\left(\Lambda^{-1}(\xi), \Lambda^{-1}(\eta)\right) .
\end{aligned}
$$

We demonstrate the procedure to calculate $w^{1}$ and thereby obtain $\bar{v}_{1}^{(2)}$. The solution to (60) for $\xi>\eta$ is

$$
w^{1}(\xi, \eta)=\int_{0}^{\eta} e^{\frac{r}{2}(\xi-\eta)} e^{r s}\left(-\frac{3 \alpha^{2}}{8 \beta}\left[1-e^{-r(s+\xi-\eta)}\right]^{2}+g(s+\xi-\eta, s)\right) d s
$$

and for $\eta \geq \xi$ the solution is

$$
w^{1}(\xi, \eta)=\int_{0}^{\xi} e^{\frac{r}{2}(\eta-\xi)} e^{r s}\left(-\frac{3 \alpha^{2}}{8 \beta}\left[1-e^{-r s}\right]^{2}+g(s, s+\eta-\xi)\right) d s .
$$

Therefore, we simply have to compute these integrals. Based on the derivatives of $v^{(1)}$, we define

$$
\begin{aligned}
G(y, k) & \triangleq \int_{0}^{y} \frac{\alpha^{2}}{4 \beta} e^{r s}\left\{\left(1-k e^{-r s}\right) \frac{e^{-r s}}{1-e^{-r s}}\left(-r s-k e^{-r s}+k\right)\right. \\
& \left.+\left(1-e^{-r s}\right) \frac{k e^{-r s}}{1-k e^{-r s}}\left(1-r s-e^{-r s}\right)\right\} d s .
\end{aligned}
$$

With the aid of this function, we have

$$
\int_{0}^{\eta} e^{r s} g(s+\xi-\eta, s) d s=G\left(\eta, \varphi_{1}\right) \quad \text { and } \quad \int_{0}^{\xi} e^{r s} g(s, s+\eta-\xi) d s=G\left(\xi, \varphi_{2}\right),
$$

where $\varphi_{1}=\exp \{-r(\xi-\eta)\}$, and $\varphi_{2}=\exp \{-r(\eta-\xi)\}$. After computing the first half of the integrals in (62) and (63) we arrive at

$$
w^{1}(\xi, \eta)= \begin{cases}e^{\frac{r}{2}(\xi-\eta)}\left[-\frac{3 \alpha^{2}}{8 \beta}\left(\frac{1}{r}\left(e^{r \eta}-1-\varphi_{1}^{2}\left(e^{-r \eta}-1\right)\right)-2 \varphi_{1} \eta\right)+G\left(\eta, \varphi_{1}\right)\right] & \text { for } \quad \xi>\eta \\ e^{\frac{r}{2}(\eta-\xi)}\left[-\frac{3 \alpha^{2}}{8 \beta}\left(\frac{1}{r}\left(e^{r \xi}-e^{-r \xi}\right)-2 \xi\right)+G\left(\xi, \varphi_{2}\right)\right] & \text { for } \quad \eta \geq \xi\end{cases}
$$


We omit the details of the tedious integrals. The remainder of the proof is to then restore the change of variables through

$$
\bar{v}_{i}^{(2)}\left(x_{1}, x_{2}\right)=e^{-\frac{r}{2}\left(\Lambda\left(x_{1}\right)+\Lambda\left(x_{2}\right)\right)} w^{i}\left(\Lambda\left(x_{1}\right), \Lambda\left(x_{2}\right)\right) .
$$

With the function $\bar{v}_{i}^{(2)}$ found above, and $v_{i}^{(2), b}$ in Appendix A, we find $v_{i}^{(2), c}=v_{i}^{(2), b}-\bar{v}_{i}^{(2)}$.

\section{References}

T. Başar and G. J. Olsder. Dynamic Noncooperative Game Theory. Academic Press, 2nd edition, 1995.

A. Bensoussan and J. Frehse. Regularity results for nonlinear elliptic systems and applications. Springer, 2002 .

J. Bertrand. Théorie mathématique de la richesse sociale. Journal des Savants, 67:499-508, 1883.

R. Cellini, L. Lambertini, and G. Ottaviano. Welfare in a differentiated oligopoly with free entry: a cautionary note. Research in Economics, 58:125-133, 2004.

A. Cournot. Recherches sur les Principes Mathématique de la Théorie des Richesses. Hachette, Paris, 1838. English translation by N.T. Bacon, published in Economic Classics, Macmillan, 1897, and reprinted in 1960 by Augustus M. Kelley.

E. Dockner, S. Jørgensen, N. v. Long, and G. Sorger. Differential games in economics and management science. Cambridge University Press, 2000.

J. Friedman. Oligopoly Theory. Cambridge University Press, 1983.

C. Harris, S. Howison, and R. Sircar. Games with exhaustible resources. SIAM J. Applied Mathematics, 70: $2556-2581,2010$.

H. Hotelling. Stability in competition. The Economic Journal, 39(153):41-57, 1929.

A. Ledvina and R. Sircar. Bertrand and Cournot competition under asymmetric costs: number of active firms in equilibrium. Submitted, 2010.

A. Ledvina and R. Sircar. Dynamic Bertrand oligopoly. Applied Mathematics and Optimization, 63:11-44, 2011.

A. Mas-Colell, M. D. Whinston, and J. R. Green. Microeconomic Theory. Oxford University Press, New York, New York, 1995.

A. Mukherjee. Price and quantity competition under free entry. Research in Economics, 59:335-344, 2005.

X. Vives. Oligopoly pricing: old ideas and new tools. MIT Press, Cambridge, MA, 1999. 